\title{
Design of Hospital Beds Center Management Information System based on HIS
}

\author{
Lu Ren ${ }^{1}$, Xiaofei Zhang ${ }^{1}$ Jingxia Wang ${ }^{1}$, Mei Sun ${ }^{1}$, Siyuan Tang ${ }^{1, a, *}$, Ni Gong ${ }^{2, b, *}$ \\ ${ }^{1}$ Xiangya Nursing School, Central South University, Changsha, China \\ ${ }^{2}$ The Third Xiangya Hospital, Central South University, Changsha, China \\ a.renlu818@126.com, b.1805278220@qq.com \\ *corresponding author
}

Keywords: Hospital Management, HIS, Bed Management

\begin{abstract}
Hospital information system(HIS) has been considered as one of the most important branch of the Medical Informatics by international academia community, with the essence of integrating all the hospital departments into a large information network to make the make the whole hospital system run better. Aiming at"difficult to be hospitalized" issues in our country, our research has designed and realized a set of safe,stable and easy-to-handle beds resource management information system according to this article HIS functional specifications. The Bed Management Information System was developed by the hospital information department, using PowerBuilder, the MVC model and the Oracle database to make the system run normally.This system has realized the bed resource management of hospitalized patients,and achieve interdisciplinary treatment for different department according to the system information,which reduce the average hospitalized stay of patients effectively.
\end{abstract}

\section{Introduction}

In the highly developed medical information environment, how to make full use of information technology to optimize the hospital's internal business processes and management processes has to be the important task for each hospital ${ }^{[1]}$. HIS has been considered as one of the most important branch of the Medical Informatics by international academia community, with the essence of integrating all the hospital departments into a large information network to make the make the whole hospital system run better.Nowadays,people'needs for health care are no longer limited to treatment,but also for prevention of disease and improvement of health process.Thus,the proportion of hospitalized patients has been increased year by year in recent years, while the patients put forward higher requirements on conditions, environment, physicians and nurses.So how to reduce the average hospitalized stay of patients as well as improve the bed turnover by doing a reasonable and scientific management of bed resourse is an urgent problem to be solved.

Many Japanese hospital started using HIS to deal information in 1980s with the rapid development and large scale ${ }^{[2]}$. They put forward the concept of HER(Electronic Health Records),which combine the EMR(Electronic Medical Records) with CPOE(Computerized Physician order entry)closely.And it means the Japanese government pays the increasingly attention on the spread of computerized method,such as Telemedicine ${ }^{[3]}$.

In our country, it has become an essential infrastructure for the operation of the hospital management business because of the widespread establishment of HIS in many advanced hospital ${ }^{[4-}$ 
${ }^{5]}$.However, literatures on hospital bed information management is extremely limited.How to combine the bed management with computerized method perfectly is still to be a problem.

\section{The Key Technologies}

\subsection{HIS}

Professor Morris. F. Collen raised the HIS with the concept of using computer and communication equipment to collect, store and process the hospital information in $1988^{[6]}$. We consider the HIS as the software system that can achieve the hospital information management for all aspect by computer in our research.The function of HIS can divided into transaction management and information services. As to transaction management, it can realize the Outpatient management, hospitalized management, medicine management, economy management and integrated information management while information services mainly used for medical,scientific research and education.

\subsection{PowerBuilder}

Started to be used in 1991 and had a peak of about 100,000 users,PowerBuilder is an integrated development environment developed by SAP. It's native data processing object is called a data window that can create, edit, and display data from a database. The object provides the programmer with tools to specify and control the appearance and behavior of the user interface, and also provides a simplified access to the contents of the database. To a certain extent, the data window allows the programmer to consider different vendors between different database management systems. Users can use multiple presentation styles to display data that can be connected to various data sources $^{[7]}$.

\subsection{MVC}

MVC is the abbreviation of "Model-View-Controller", MVC application program is always composed of these three parts. Event causes Controller to change Model or View, or both. As long as the Controller changes the Models data or properties, all Views dependent on it will be automatically updated. Similarly, as long as the Controller changed the View, View will get the data from the potential of Model to refresh themselves ${ }^{[8]}$. View is on behalf of the user interface, for Web applications, it can be summarized as the HTML interface, but it is possible be XHTML, XML and Applet $^{[9]}$. With the complexity and scalability of the application, the processing of the interface also becomes a challenge. An application may contain a lot of different views, the MVC design pattern for view processing is limited to data acquisition and processing on the view, as well as the user's request, but don't cover the business process on the view. Then, Business process will be handled by Model. Model is the process of business process / state and the development of business rules. The design of business model is something of the core of MVC. MVC design pattern tells us that if we want extract the application of the model according a certain rule, the level of extraction is very important, which can be design basis for judging whether the developer is excellent .The controller can be understood as receiving requests from the user, then match the model with the view, and complete the user's request together.

\subsection{Oracle Database}

Oracle database, often called Oracle RDBMS or Oracle,is a object relational database management system and produced and sold by Oracle Corporation.It keep tracks of data storage in 
computer as well as SYSTEM tablespace. The SYSTEM tablespace contains data dictionaries (default) metrics and clusters, where the data dictionary is contained by a specialized collection table that contains information about all user objects in the database ${ }^{[10]}$.

\section{System Requirement Analysis}

\subsection{The overall requirement analysis}

It is important to establish a normative, safe and stable, simple bed resource management system based on the demand of patients,users and leaderships in hospital.The overall requirement analysis was showed in figure 1.

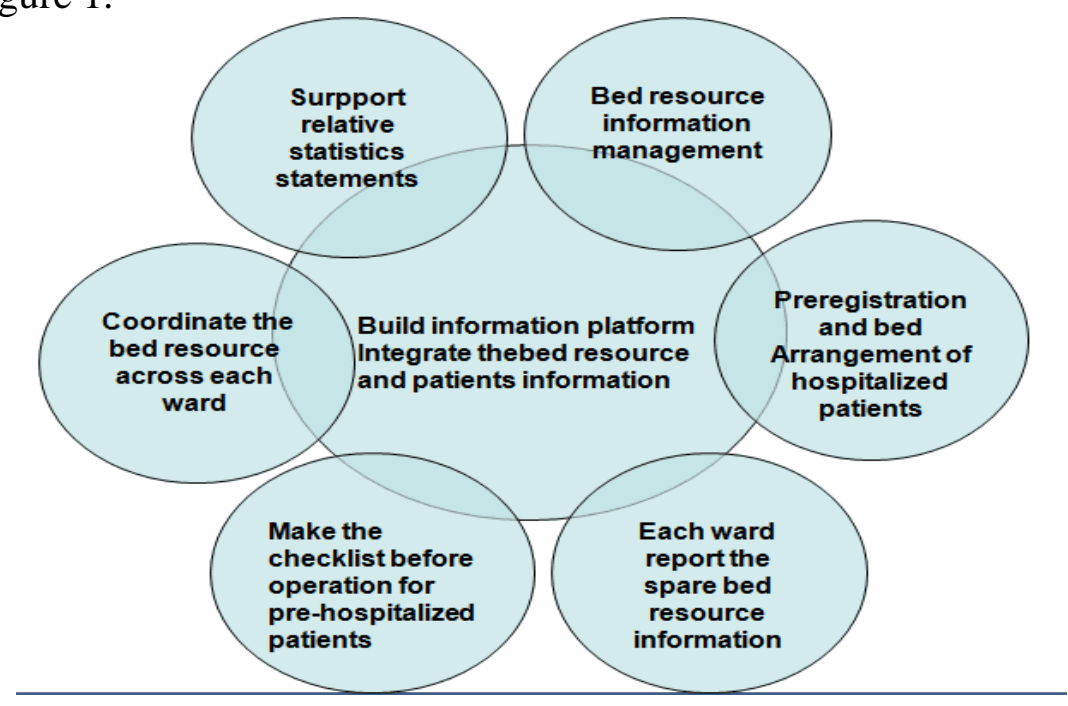

Figure 1: Beds Center Management Information System overall requirement analysis.

\subsection{The functional requirement analysis}

The functional requirement analysis including the following 5 aspects:

1) Bed appointment management;

2) Bed report management;

3) The overall bed list in depatment;

4) Appointment and transfer between different depatment;

5) System settings.

\subsection{The non-functional requirement analysis}

The non-functional requirement analysis can divided into 4 aspects:

1) Accuracy and timeliness in system processing

2) Safety of system

3) Scalability of system 
4) Maintainability of system

\subsection{Feasibility Analysis}

The feasibility analysis contains business feasibility analysis and technical feasibility analysis.

\section{System Intergrated Design}

The overall structure of hospital management information system is divided into the execution environment layer, data layer, application support layer, business logic layer and presentation layer.At the same time,the information system also abide by the relevant policies and regulations, professional standards as well as the security support and system operation and maintenance support in the construction process. The overall system structure shows in diagram 2.In the overall system structur,our research mainly design and implementate Hospital Beds Center Management, whose main task is to allocate, replace beds for patients while evacuate beds when patients are transferred or discharged.Details were showed in figure 2.

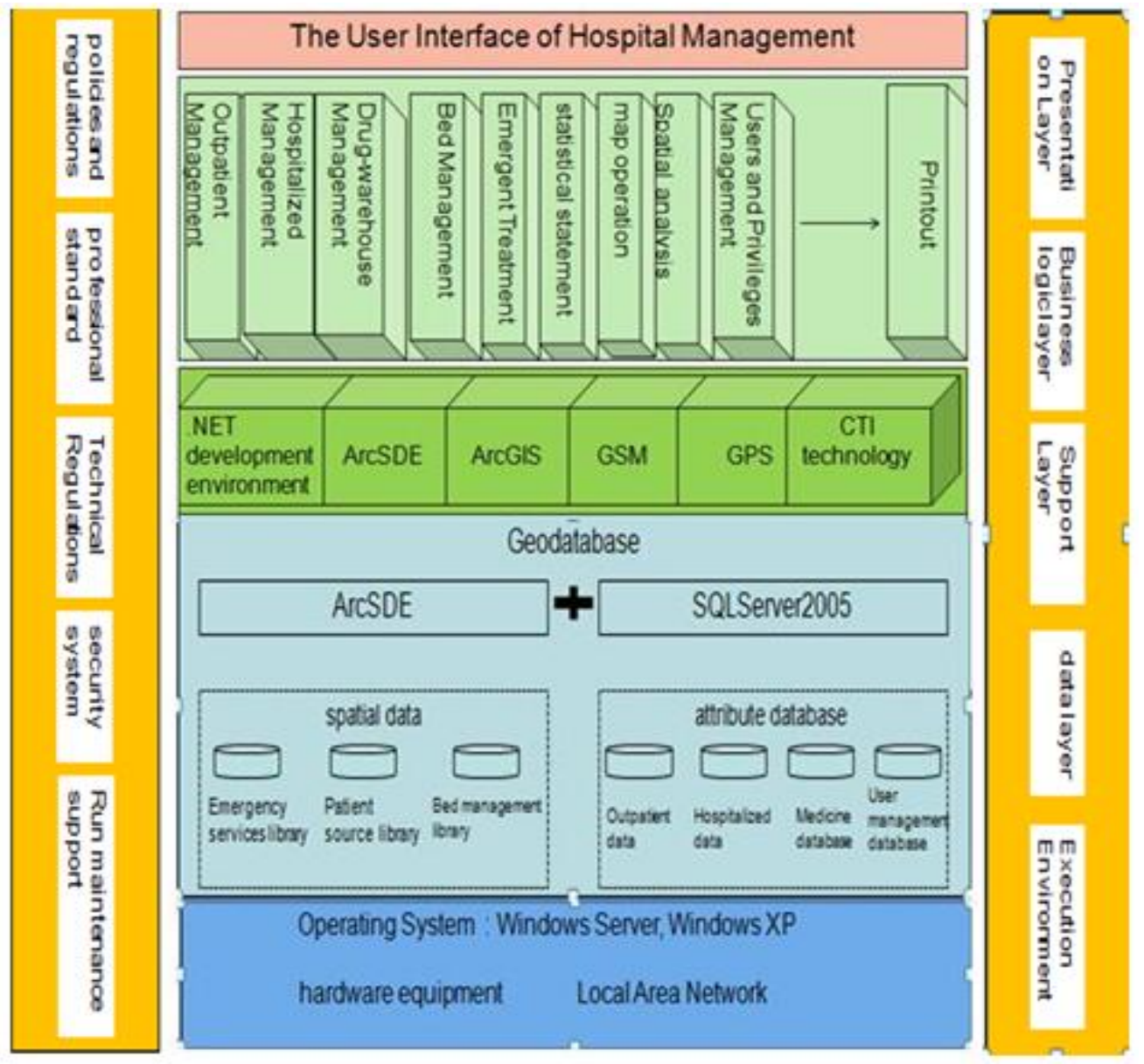

Figure 2. The integrated system structure. 


\section{System Module Implementation}

\subsection{Bed reservation management module implementation}

This module can realize the following functions:

1) Reserved record;

2) Scheduled record;

3) Canceled Records;

4) Hospitalized Records.

\subsection{Bed report management}

\subsection{List of bed in ward}

1) The whole bed information of hospital;

2) The emergency bed list.

\subsection{Appointment transfer branch management}

\subsection{System settings}

6. System Test

\subsection{Functional test}

The test testers are composed of developers who are responsible for developing the corresponding modules, and then perform the functional tests overlapping after performing unit tests.

\subsection{Pressure test}

Our research chose 5 bed number in the waed as examples to perform the pressure test.The result showed in table 1.

Table 1. Operation Response Time Test

\begin{tabular}{cccccc}
\hline Bed Number & T1 & T2 & T3 & T4 & T5 \\
\hline Test $\mathbf{1}(\mathbf{m s})$ & 3165 & 7652 & 2647 & 8664 & 9314 \\
Test $\mathbf{( m s )}$ & 6464 & 2456 & 3654 & 3246 & 6723 \\
Test 3 (ms) & 3464 & 3665 & 6794 & 7321 & 1546 \\
Test 4 (ms) & 9841 & 6546 & 3264 & 6846 & 1646 \\
Test 5 (ms) & 8732 & 2648 & 2156 & 8633 & 6467 \\
Average Time (ms) & 6333 & 4593 & 3703 & 6942 & 4899 \\
\hline
\end{tabular}




\section{Conclusion}

The overall architecture and function flow of the system are analyzed and introduced in detail by using the overall system architecture diagram and the bed center function flow chart in this paper.We also present the three-tier structure of presentation layer, business logic layer and data layer respectively in details. According to the HIS function requirement as well as combining the intrinsic HIS preferably,our research has designed and realized a set of safe,stable and easy-tohandle beds resource management information system aming at the problems of "difficult to be hospitalized", which provide the hospital beds centralized management with comprehensive information solutions ${ }^{[11]}$.Although the hospital's bed management mode was optimized and the average length of hospital stay was reduced, the problem of hospitalization was relieved to a great extent ${ }^{[12]}$. However, how to protect the quality of patients under the premise of the quality of care, making the average hospital stay in patients with a more reasonable arrangement, still need a better communication between hospital management and patient, to complete a more perfect bed management.

\section{Acknowledgment}

We thank the Central South University for the support of the Fundamental Research Funds for the Central Universities of Central South University under grant number 2015zzts107,the the support of the National Natural Science Foundation of China under grant number 81370974 and the support of CMB Nursing Young Teachers Research Project under grant number 13-168-201606. Corresponding author Siyuan Tang and Ni Gong share the equally contribution in this paper.

\section{Reference}

[1] Pinggen,W., and Yunsuo,G. (2012)Preliminary Study on the Distribution Plan of Hospital Beds in Large General Hospital.Chinese Journal of Hospital Statistics,13,7-8.

[2] Schneeweiss,S. (2014)Learning from big health care data.N Engl J Med, 370,2161-2163.

[3] Parkinson,R.C.(2014)Tying supply chain costs to patient care. Healthc Financ Manage,68,42-45.

[4] Ge,Y.J.,Xiong.W.(2010)Disscusion on Hospital Bed Management.Journal of Suzhou University (Philosopy and Social Science),18,.49-51.

[5] Chen,Y.(2010) Present Situation and Countermeasure of Hospital Bed Management.Management Observation,14,154-155.

[6] Li,H.L.(2014)Research and Design of Enterprise Management Improvement Project Management Platform Based on JMS.Tianjin University.

[7] Microsoft Corporation, ASP.NET MVC: The Official Microsoft ASP.NET Site[EB/OL], http://www.asp.net/mvc2012-2.

[8] Scott Klein.(2010)Pro Entity Framework 4.0.

[9] Ku,H.S.,Kim,S. and Kim,H.(2014)DialysisNet:Application for Integrating and Management Data Sources of Hemodialysis Information by Continuity of Care Record.Healthc Inform Res, 20, 145-151.

[10] Nguyen,J.M.,Six,P.and Antonioli,D.(2015)A simple method to optimize hospital beds capacity.Journal of Health Geographies,74,39-49.

[11] Utley,M., Gallivan,S.and Davis,K.(2013)Worrall Estimating bed requirements for an intermediate care facility.Eur. J. Op, 92-100.

[12] Jun,Y. and Cheng,C.(2014) Design of system architecture based on J2EE workflow platform.Electronic technology and software engineering, 11,59-60. 